\title{
Influencia de los hábitos de estudio en la elección de carreras profesionales en alumnos del cuarto y quinto grado de secundaria
}

\author{
Influence of the study habits in the choice of professionals careers in fourth and fifth \\ grade of secondary education
}

\author{
Víctor Elías Ríos Cubas ', Luz María Supo Zapata ${ }^{2}$ \\ Universidad Continental
}

\section{RESUMEN}

Objetivos: Determinar la influencia de los hábitos de estudio en la elección de carreras profesionales en alumnos de los últimos dos grados, $4^{\circ}$ y $5^{\circ}$ de educación secundaria. Métodos: El estudio es de alcance descriptivo - correlacional, con un diseño transversal. En la recolección de datos se utilizó dos instrumentos: el inventario de hábitos de estudio y el inventario de intereses vocacionales. La población ha estado constituida por un total de 6901 alumnos de colegios estatales y privados, entre varones y mujeres de la ciudad de Huancayo - Perú. De los 6 901 alumnos evaluados se han tomado 3 953, ya que 1528 fueron incompatibles y 1420 en blanco. El tamaño de la población obedece a un estudio de tipo censal. Resultados: Se ha encontrado que el $91 \%(n=3599)$ de los estudiantes tienen deficientes - regulares hábitos de estudio, siendo los varones $(93,3 \%) q u i e n e s$ tienen más dificultades en relación a las mujeres $(89,1 \%)$, por tanto se evidenció la relación entre los hábitos de estudio y el género de los estudiantes. Se mostraron preferencias vocacionales profesionales en mujeres hacia carreras que corresponde al manejo de cifras $(21,9 \%)$, y en el caso de varones las preferencias vocacionales se relacionaron a carreras orientadas a la organización de grupos y a la búsqueda de beneficios comunes $(21,5 \%)$. Por otro lado aquellas carreras relacionadas a labores de oficina tuvieron las más bajas puntuaciones $(0,1 \%)$. La tendencia de los alumnos está dirigida a carreras que tienen relación con la creatividad (12,3\%). Conclusiones: Existe incidencia de los hábitos de estudios en los

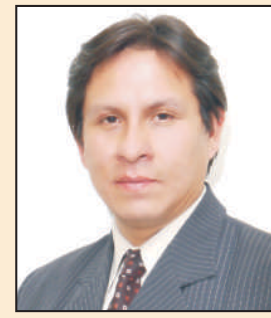

Víctor Ríos

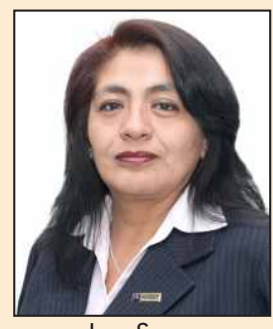

Luz Supo

intereses vocacionales profesionales y podrían presentarse dificultades de vocación profesional y de futura incompatibilidad laboral.

Palabras clave: Influencia, hábitos, estudio, elección, orientación vocacional.

1 Lic. en Psicología, Mg. en Recursos Humanos, Mg. en Desarrollo de habilidades y competencias, Candidato a Doctor en Psicología, Consultor Senior y Gerente General de la Consultora "Liderando Kambios".

2 Lic. en Estadística, Candidata a Magister en Administración de Negocios, Docente Investigadora de la Universidad Continental. 


\section{ABSTRACT}

Objectives: Determine the influence of the study habits in the choice of professional careers in students of the last two grades, fourth and fifth grade of secondary education. Methods: The study has correlation and descriptive scope, with a cross sectional design. In the data collection was used two instruments: the inventory of study habits and the inventory of vocational interests. The population has been formed by a total of 6901 students of private and public high schools, between men and women of Huancayo city, Peru. From the 6901 students evaluated, have been taken 3953 , since 1 528 were incompatible and 1420 on blank. The population size follows a census study type. Results: Has been found that 91\% ( $n=$ 3 599) of the students have poor or regular study habits, been men $(93,3 \%)$ who have more difficulties in relation to women $(89,1 \%)$. Therefore, the relationship is evidenced between the study habits and the students gender. It shows vocational professional preferences in women toward carriers that correspond to the management of numbers $(21,9 \%)$ and in the case of men the vocational preferences are related to carriers oriented to the organization of groups and to the search of common benefits $(21,5 \%)$. On the other hand, carriers related to office labor had the lowest scores $(0,1 \%)$. The student trend is directed to carriers that have relation with creativity (12,3\%). Conclusions: Exists incidence of study habits in the vocational professional preferences considering that there might be difficulties of professional vocation and a future labor incompatibility.

Key words: Influence, habits, study, selection, vocational orientation.

\section{INTRODUCCIÓN}

Este estudio se justifica por el bajo rendimiento académico, deserción y alta rotación en la elección de una carrera profesional. La finalización de la escolaridad marca el inicio de la transición al llamado mundo adulto representados por dispositivos a nivel familiar, social y educativo, entonces podemos advertir que el problema de elección vocacional se encuentra diferenciada, sin embargo es un denominador común la toma decisiones rápidas de forma insegura. Entonces es vital que los jóvenes antes de tomar una decisión vocacional puedan tener conocimiento de las características del trabajo profesional que implica dicha carrera, así como tener un panorama amplio (1), real y actualizado de las mismas. Siendo importante que los jóvenes se informen de los planes de estudios, la exigencia académica, las materias principales y el método de estudio apropiado para aprender la carrera elegida. (2)

En este contexto podemos afirmar que la historia vocacional se va construyendo junto con la historia personal en un proceso orientado hacia el futuro (3); desarrollando un modelo de soporte vocacional en la cual el docente tutor asuma su rol desde la perspectiva académica (4) que implica el reforzamiento de métodos de estudios prácticos, desarrollo de pensamiento crítico y estilos de aprendizaje, y desde la perspectiva humana relacionada a la construcción de procesos de identidad social y psicológica direccionados a la estructuración de sus planes, metas, intereses y expectativas futuras (5). Dicho lo anterior podemos afirmar que el docente tutor tiene como objetivo primordial reforzar aptitudes logradas por los jóvenes (6) y la generación de la responsabilidad y compromiso con sus proyectos (7), ayudándoles a reconocer sus fortalezas y obstáculos para propiciar su autonomía. (8)

La situación problemática se manifiesta por una carencia de aplicación de técnicas y hábitos de estudio en las carreras profesionales por lo que no solo genera inconsistencias en la permanencia en la carrera, sino también afecta su rendimiento académico. Lo cual afectaría a sus futuras competencias laborales, ya que los 
profesionales de las diferentes carreras posteriormente deberán generar una sostenibilidad académica en su desempeño laboral.

Aproximándonos al desarrollo de nuestro tema, observamos que uno de los escollos a sortear por las instituciones de educación superior es la deficiente preparación académica, los problemas de adaptación y falta de organización en el estudio de los jóvenes egresados de la enseñanza media (9). Aún más, se advierte una clara desorientación en la información aislada y superficial (provocando elecciones basadas en impulsos, costumbres o intereses de estatus), y dificultad en sus aptitudes para asimilar contenidos debido a la ausencia de métodos y el uso correcto de técnicas de estudio (8). En este escenario la posibilidad de fracaso y deserción de las carreras profesionales elegidas es obvio; existiendo una tendencia mayor de deserción en varones. (9)

Nuestro principal objetivo es determinar la influencia de los hábitos de estudio en la elección de carreras profesionales en alumnos de los últimos dos grados, $4^{\circ}$ y $5^{\circ}$ de educación secundaria. Planteándonos como hipótesis la existencia de la influencia del tipo de colegio (particular o estatal) de donde provengan los alumnos con sus hábitos de estudio, y la diferencia en los hábitos de estudio según el género de los mismos. Tomando la segunda perspectiva desarrollada que se relaciona a la ausencia de métodos de estudios, sobre todo en los aspectos que se relacionan al manejo de técnicas de estudio que refuercen los logros alcanzados y que posteriormente se constituyan en instrumentos de autovalidación de su aprendizaje y que puedan generar hábitos de estudio automáticos para el logro de sus objetivos de modo efectivo. (10)

Consideramos los autores que no se deben confundir los hábitos (prácticas constantes de las mismas actividades) con las técnicas (procedimientos o recursos). No obstante unas y otras, empero, coadyuvan a la eficacia del estudio. De un lado, el hábito de estudiar es necesario si se quiere progresar en el aprendizaje. De otro, conviene sacar el máximo provecho a la energía que requiere la práctica consciente e intensiva del estudio por medio de unas técnicas adecuadas. En este contexto las aptitudes del joven desempeñan un papel importante ya que tienen que ver con un conjunto de características consideradas como habilidades que deben ser reforzadas y desarrolladas adecuadamente para el logro de objetivos personales de su futuro profesional. (11)

Entonces se evidencia la conjugación del joven alumno, el docente- tutor y el propio entorno a través de dos teorías: 1). La teoría de los procesos motivacionales las cuales influyen en la motivación de los jóvenes alumnos, a través de sus motivaciones externas (psicología conductual) y sus motivaciones internas (psicología cognitiva); además de una adecuada fijación de objetivos relacionados a sus expectativas de los jóvenes. 2). La teoría de la atribución la cual se halla relacionada a las motivaciones descritas anteriormente, siendo éstas quienes movilizan emociones intensas $y$ promueven un desempeño académico. Ambas teorías promueven el manejo de una metodología de las técnicas de estudios las cuales desarrollen habilidades de los jóvenes, de acuerdo al tipo de reforzamiento que los lleve al éxito o fracaso. (12)

Podemos afirmar la importancia de la enseñanza de habilidades de estudio en la cual se hace necesario trabajar sus motivaciones, aptitudes, además de una apropiada intervención profesional adulta en el contexto que rodea a los jóvenes. No obstante se debe poner énfasis en indicadores medibles y comparables de técnicas de estudio: la gestión de esfuerzo y tensiones, el manejo del tiempo, la toma de notas en clases, la lectura de textos, las pruebas, y la investigación bibliográfica. Sin embargo, se tiende a elegir carreras que "supuestamente" implican menos esfuerzo.

Aún más, observamos que existe desinterés por aquellas carreras relacionadas a labores de oficina porque serian rutinarias y 
a pesar que sean poco exigentes, sin que ello implique para su formación la afluencia de mayores hábitos y métodos de estudio, igualmente no son de su preferencia (13).

\section{MATERIAL Y METODOS}

Se aplicaron dos instrumentos colectivos en aulas de acuerdo a previa coordinación con los colegios particulares. Se desarrollaron formatos en fichas ópticas para facilitar el procesamiento de datos. Los instrumentos fueron los siguientes: 1). El inventario de intereses vocacionales de Galilea Pagola que describe más de 50 tareas y trabajos, a las que el usuario debe responder si las elige o no para desempeñarlas a corto y/o medio plazo; 2). El Inventario de hábitos y Estrategias de Edelvives, el cual consiste en 64 declaraciones agrupadas en dos dimensiones: Organización de estudios (lugar de estudio, planificación.) y estrategias de aprendizaje (procesos de estudio, lecturas, tomar apuntes, resumir textos, preparar exámenes, realizar trabajos escritos), las cuales la persona debe contestar falso o verdadero. Cada instrumento de medición tiene un protocolo de preguntas y una hoja de respuestas, se utilizó fichas ópticas para facilitar su procesamiento.

La aplicación de los instrumentos se realizó a un total de 6901 alumnos del $4^{\circ}$ grado y $5^{\circ}$ grado de educación secundaria en Huancayo- Perú; en forma colectiva en sus respectivas aulas (a todas las secciones a la vez de 4to y 5to de un colegio), previa coordinación, para ingresar a las aulas, con los directores de los colegios evaluados. Luego se ingresaron los resultados al sistema automatizado con el apoyo del escáner de lectura de tarjetas ópticas. Posteriormente se procesaron los resultados aplicando la estadística descriptiva y correlacional para analizar los intereses vocacionales y los hábitos de estudio.

\section{RESULTADOS}

Se evaluaron un total de 6901 alumnos entre varones y mujeres de colegios particulares y colegios estatales. De la población inicial, se consideraron 3953

Tabla № 1: Carreras profesionales por áreas de interés

\begin{tabular}{|c|c|c|}
\hline A & Manejo de cifras & $\begin{array}{l}\text { Contabilidad, economía, estadística, matemática, investigación, administración } \\
\text { bancaria, computación, ingeniería (electrónica y de sistemas) }\end{array}$ \\
\hline B & $\begin{array}{l}\text { Investigar razón } \\
\text { de hechos }\end{array}$ & $\begin{array}{l}\text { Física, química, farmacia, meteorología, geofísica, antropología, arqueología, } \\
\text { medicina, odontología, psicología, enfermería, nutrición humana, laboratorio } \\
\text { técnico, tecnología médica. }\end{array}$ \\
\hline C & $\begin{array}{l}\text { Manejo de cosas } \\
\text { reales y tangibles }\end{array}$ & $\begin{array}{l}\text { Ingenierías (civil, eléctrica, mecánica), mecánica automotriz, técnico en } \\
\text { computación. }\end{array}$ \\
\hline D & $\begin{array}{l}\text { Orientar, educar } \\
\text { o guiar }\end{array}$ & $\begin{array}{l}\text { Psicología, educación, nutrición humana, enfermería, medicina, obstetricia, } \\
\text { trabajo social, historia, turismo, derecho. }\end{array}$ \\
\hline$E$ & Comunicación & $\begin{array}{l}\text { Literatura, lingüística, periodismo, ciencias de la comunicación, publicidad, } \\
\text { historia, derecho, traducción. }\end{array}$ \\
\hline $\mathrm{F}$ & $\begin{array}{l}\text { Organizar grupos } \\
\text { y buscar beneficios } \\
\text { comunes }\end{array}$ & Administración, sociología, diplomacia, institutos armados y policiales. \\
\hline G & Creatividad & $\begin{array}{l}\text { Arquitectura, dibujo, diseño (grafico, publicitario), publicidad, artes plásticas, } \\
\text { fotografía. }\end{array}$ \\
\hline $\mathrm{H}$ & Labores de oficina & Traducción, bibliotecología, secretariado ejecutivo, asistente administrativo. \\
\hline
\end{tabular}


Tabla № 2: Dsitribución de estudiantes evaluados por hábitos de estudio y resultado de test vocacional

\begin{tabular}{|c|c|c|c|c|c|c|c|c|c|c|c|}
\hline \multicolumn{12}{|c|}{ Resultado Test Vocacional } \\
\hline & & & A & B & $C$ & D & $E$ & $\mathrm{~F}$ & G & $\mathrm{H}$ & Total \\
\hline \multirow{12}{*}{ 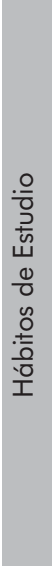 } & Deficiente & $N^{\circ}$ Eval. & 641 & 248 & 259 & 418 & 430 & 692 & 414 & 2 & 3104 \\
\hline & & $\%$ Eval. & $16,2 \%$ & $6,3 \%$ & $6,6 \%$ & $10,6 \%$ & $10,9 \%$ & $17,5 \%$ & $10,5 \%$ & $0,1 \%$ & $78,5 \%$ \\
\hline & Regular & $N^{\circ}$ Eval. & 125 & 53 & 33 & 78 & 70 & 98 & 38 & 0 & 495 \\
\hline & & $\%$ Eval. & $3,2 \%$ & $1,3 \%$ & $0,8 \%$ & $2,0 \%$ & $1,8 \%$ & $2,5 \%$ & $1,0 \%$ & $0,0 \%$ & $12,5 \%$ \\
\hline & Bueno & № Eval. & 77 & 28 & 10 & 41 & 36 & 40 & 27 & 0 & 259 \\
\hline & & $\%$ Eval. & $1,9 \%$ & $0,7 \%$ & $0,3 \%$ & $1,0 \%$ & $0,9 \%$ & $1,0 \%$ & $0,7 \%$ & $0,0 \%$ & $6,6 \%$ \\
\hline & Muy bueno & № Eval. & 21 & 6 & 5 & 12 & 10 & 16 & 9 & 0 & 79 \\
\hline & & \% Eval. & $0,5 \%$ & $0,2 \%$ & $0,1 \%$ & $0,3 \%$ & $0,3 \%$ & $0,4 \%$ & $0,2 \%$ & $0,0 \%$ & $2,0 \%$ \\
\hline & Excelente & $N^{\circ}$ Eval. & 1 & 2 & 4 & 3 & 3 & 3 & 0 & 0 & 16 \\
\hline & & \% Eval. & $0,0 \%$ & $0,1 \%$ & $0,1 \%$ & $0,1 \%$ & $0,1 \%$ & $0,1 \%$ & $0,0 \%$ & $0,0 \%$ & $0,4 \%$ \\
\hline & Total & $N^{\circ}$ Eval. & 865 & 337 & 311 & 552 & 549 & 849 & 488 & 2 & 3953 \\
\hline & & \% Eval. & $21,9 \%$ & $8,5 \%$ & $7,9 \%$ & $14,0 \%$ & $13,9 \%$ & $21,5 \%$ & $12,3 \%$ & $0,1 \%$ & $100,0 \%$ \\
\hline
\end{tabular}

alumnos, para la presente investigación, ya que $1528(22,1 \%)$ fueron considerados incompatibles y $1420(20,6 \%)$ fueron considerados en blanco. De la población seleccionada para la investigación de 3953 alumnos, el $54,9 \%$ fueron mujeres y el $45,1 \%$ fueron varones, de 4 to y 5 to de secundaria de 88 colegios. Donde el 63,3\% de los estudiantes son de colegios estatales y el $36,7 \%$ son de colegios particulares.

Es preocupante determinar que el $91,0 \%$ de los alumnos tienen deficientes o regulares hábitos de estudios. En tanto que sólo el $9,0 \%$ de todos los evaluados muestran hábitos de bueno a excelentes ( siendo $9,6 \%$ de colegios particulares y $8,6 \%$ de Estatales). Se determinó además, mediante la prueba no paramétrica de Chi cuadrado, al 5\% de nivel de significancia que no existe relación entre los hábitos de estudio y el tipo de colegio

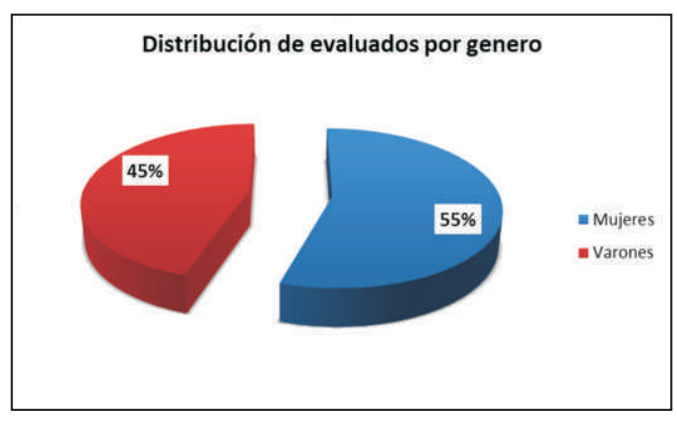

Figura 1. Evaluados por genero (particular o estatal) de donde provengan los estudiantes; sin embargo si existe relación entre los hábitos de estudio y el género. Con respecto a los hábitos de estudio y el género, se encontró que los varones (93,3\%) muestran más deficiencias en los hábitos de estudios que las mujeres $(89,1 \%)$.

De acuerdo a los resultados obtenidos en el test vocacional, tenemos que hay mayor preferencia $(21,9 \%)$, sobre todo en mujeres, por el área vocacional $A$ : Interés por trabajos que involucren el manejo de cifras. $Y$ en segundo lugar $(21,5 \%)$, sobre todo en varones, quienes eligen el área vocacional $F$, donde existe interés por organizar a personas 0 grupos de personas, para obtener posteriormente un beneficio También podemos advertir que la tendencia de los alumnos es orientarse al área vocacional $G(12,3 \%)$, manifestando interés

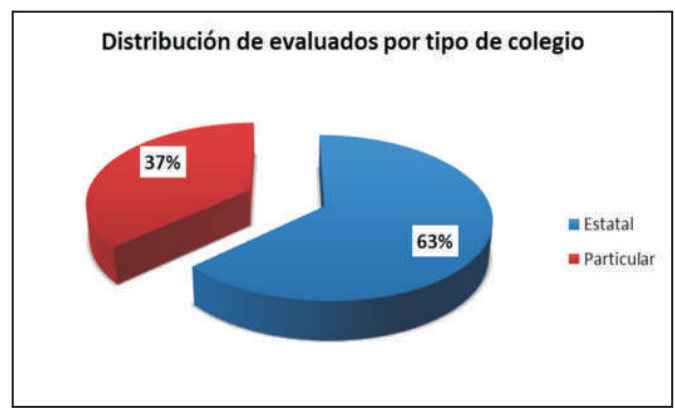

Figura 2. Evaluados por tipo de colegio 
por la creatividad. Por último y con muy poco preferencia $(0,1 \%)$ por el área vocacional $\mathrm{H}$, en la cual se advierte interés en las labores de oficina. (Tabla $\mathrm{N}^{\circ} 1$ y Tabla $\mathrm{N}^{\circ} 2$ ).

No existe relación entre las áreas vocacionales y el tipo de colegios (estatales y privados), sin embargo existe relación entre las áreas vocacionales y el género. Con la prueba de hipótesis de chi cuadrado se contrastó que existe relación entre el área de vocación obtenida en el test vocacional y los hábitos de estudio (5\% de nivel de significancia).

\section{DISCUSIÓN}

Se afirma que la elección de carreras profesionales de los estudiantes tiene como variable de gran utilidad el desarrollo de los hábitos de estudio; insumo importante para la construcción de su aprendizaje. En este contexto, de acuerdo a los resultados analizados encontramos que tanto las decisiones vocacionales en relación al desarrollo de hábitos de estudio es igual tanto para colegios particulares como para colegios estatales (chi-cuadrado $=0,4$ ); de lo cual advertimos que todos los jóvenes independientemente del tipo de colegio se encuentran en una transición de la personalidad, y de toma de decisiones certeras de su futuro. Sin embargo, uno de los aspectos vitales como lo mencionan Ferrari (1) y Goldberg (2), es que los jóvenes deben tener un proceso de orientación vocacional profesional real, concreto y adaptado a la etapa evolutiva del joven, lo cual permita que las instituciones de educación superior puedan colaborar a mejorar los problemas de adaptación y falta de organización en el estudio que presentan los jóvenes que egresan de la enseñanza media (9). En este sentido también determinamos la idea que los jóvenes deben tener una orientación vocacional profesional real, adecuada y concreta. (10) Además deben informarse acerca del estilo de vida (1) de la futura carrera profesional, los planes de estudios, la exigencia académica, y sobretodo el método de estudio apropiado para confrontar la carrera elegida. (2)

Encontramos que los jóvenes adolescentes presentan dificultades de aptitud, porque no existe ausencia de métodos y uso correcto de técnicas de estudio que promuevan hábitos de estudios adecuados a la exigencia de la futura carrera profesional ( $n=3599$ que corresponde al 91\%); en este sentido los planteamientos teóricos reafirman esta conclusión en la cual se enuncia que los jóvenes deben asimilar las distintas formas de incorporar conocimientos y procedimientos (8) son necesarios para su desarrollo profesional (10). Por otro lado las mujeres desarrollan mejores hábitos de estudios $(10,9 \%)$ a diferencia de los varones quienes, si, presentan dificultades en desarrollar hábitos de estudios (6,7\%); por lo cual la posibilidad de fracaso y/o deserción de las carreras profesionales tendría una tendencia desfavorable para los varones (9). De modo coincidente aquellas carreras que tuvieron mayor puntuación en el Área A de manejo de cifras $(24,7 \%)$, y el Área $F$ relacionada a la organización de grupos y la búsqueda de objetivos comunes $(23,6 \%$ ), son aquellas que presentan hábitos de estudios inadecuados (deficiente y regular); siendo el $19,4 \%$ en el Área $\mathrm{A}$ y el $20 \%$ en el Área $\mathrm{F}$ que poseen hábitos de estudio deficiente, haciendo un total del $39,4 \%$. Lo cual sugerimos a promover líneas de investigación que corroboren el rendimiento académico de las carreras profesionales mencionadas, y aún más cotejar la satisfacción laboral y desarrollo profesional de aquellas personas que habrían elegido estas carreras para identificar el desarrollo adecuado de las competencias profesionales y la adaptabilidad al perfil de las competencias laborales que exigirá toda organización.

Por otro lado, se observa en la investigación realizada que los varones y las mujeres de colegios particulares y estatales muestran preferencia casi nula ( $\mathrm{n}=2$ que corresponde al $0,1 \%)$ por el Área $\mathrm{H}$ relacionada a las labores de oficina porque serian rutinarias; dato que sería relevante y coincidente con la 
investigación realizada por Ríos (13). Esto nos haría presuponer que aquellas carreras técnicas que no le den dinamismo y consistencia en su utilidad podrían tener dificultades de permanecer en el mercado educativo, aún más se podría visualizar que las carreras técnicas en muchos de los casos serían asumidas solo como una decisión pasajera, ya que la perspectiva de los jóvenes sería los estudios superiores universitarios. Entonces afirmamos que los jóvenes tienen muchas aspiraciones y sueños de cumplir metas personales y profesionales (13), sin embargo aquellos deben realizar un proceso de autoanálisis para advertir sus reales habilidades, en insumos básicos (aptitud, motivación, métodos y hábitos de estudios), los cuales deben ser reforzados en el tiempo desde lo técnico- académico y desde lo emocional humano. Y no deben llevarse por pensamientos ilusorios que siguiendo carreras orientadas a la creatividad no existe una exigencia académica adecuada al desarrollo profesional.

En conclusión la elección del futuro implica un proceso complejo en el cual intervienen varios actores (el apoyo y reforzamiento de la familia, la influencia social, el contexto económico, el propio proceso de cambios del joven adolescente y el docente tutor). Siguiendo a Fry, López y Gall tenemos que los jóvenes, que no han generado la responsabilidad y compromiso con sus proyectos, y la falta de reconocimiento de fortalezas pueden generar la no inserción de los hábitos de estudio en actitudes sencillas de su vida cotidiana $(5,7,8)$. Para esto, es vital reforzar las aptitudes logradas por los jóvenes desde épocas tempranas, ya que muchas habilidades aparecen en la infancia y éstas pasan desapercibidas o son reforzadas inadecuadamente por lo que la tendencia sería su desaparición (6). Entonces podemos resaltar la importancia de uno de los actores del proceso vocacional que es el docente tutor, el cual debe asumir su rol desde la perspectiva académica para promover métodos de estudios útiles, prácticos y significativos; desarrollar el pensamiento crítico y estilos de aprendizaje. (3) Y asumir su rol desde la perspectiva humana en el cual coadyuve en la construcción de procesos de identidad social y psicológica de los jóvenes; promueva la reflexión y direccionamiento de sus planes, metas, intereses y expectativas personales y futuras (4). 


\section{REFERENCIAS BIBLIOGRÁFICAS}

1. Ferrari L. Como elegir una carrera. Buenos Aires: Planeta; 1995.

2. Goldberg B. ¿̇Qué quiero ser? Los adolescentes y la vocación. Buenos Aires: El ateneo; 2002.

3. Rascován S. Orientación Vocacional. Aportes para la formación de orientadores. Buenos Aires: Novedades Educativas; 1998.

4. Gaskins I, Thorne E.Cómo enseñar estrategias cognitivas en la escuela. El manual Benchmark para docentes. Buenos Aires: Paidós Educador; 1999.

5. Fry R. Cómo estudiar. México: Artgraph; 2009.

6. Aisenson D Colb. Aprendizaje, sujetos y escenarios. Investigaciones y prácticas en psicología educacional. Buenos Aires: Novedades Educativas; 2007.

7. López A. La orientación vocacional como proceso. Teoría, técnica y práctica. Buenos Aires: Bonum; 2003.

8. Gall M,Gall J. Jacobsen D,Bullock T. Herramientas para el aprendizaje. Buenos Aires: Aique grupo editor; 1990.

9. Rascován S. Orientación vocacional. Una perspectiva crítica. Buenos Aires: Paidós; 2005.

10.Albaladejo N. La acción tutorial. Alicante: Disgrafos; 1992.

11. Martínez V, Pérez $O$, Torres $L$. Análisis de los hábitos de estudio en una muestra de alumnos universitarios; Madrid: Revista; 2005.

12.Arnaiz, Isus S. La tutoría, organización y tareas. Barcelona: Biblioteca del aula; 1995.

13. Ríos V. Estilos de personalidad en los procesos de orientación vocacional en alumnos del cuarto y quinto grado de secundaria: Apuntes de ciencia \&Sociedad. Universidad Continental. $2011 ; 1(1): 3-8$.

Correo electrónico:

vrios@continental.edu.pe

Isupo@continental.edu.pe 